\title{
El diseño como estrategia de circularidad en el aprovechamiento de residuos agroindustriales ${ }^{1}$
}

\author{
The design as a circular economy strategy for the utilization of \\ agroindustrial residues
}

Cómo citar:

Buraglia Osorio, M., 2021. El diseño como estrategia de circularidad en el aprovechamiento de residuos agroindustriales. Designia, 8(2), 131-151.

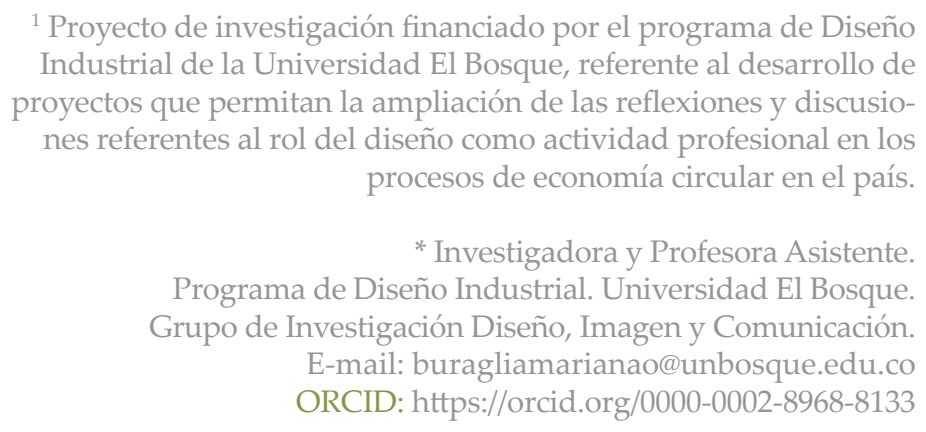

${ }^{1}$ Proyecto de investigación financiado por el programa de Diseño royectos que permitan la ampliación de las reflexiones y discusioprocesos de economía circular en el país.

* Investigadora y Profesora Asistente. Programa de Diseño Industrial. Universidad El Bosque. E-mail: buragliamarianao@unbosque.edu.co ORCID: https://orcid.org/0000-0002-8968-8133

\section{Palabras clave:}

Economía circular, residuos agroindustriales, materiales biocompuestos, materiales alternativos, ecodiseño, consumo sostenible, producción sostenible

Key words:

Circular economy, agricultural wastes, composite materials, new materials, eco-design, sustainable consumption, sustainable production

Recibido: 20/11/2020 Aceptado: 18/12/2020

\section{Resumen:}

Las políticas de implementación de economía circular en Colombia han priorizado el aprovechamiento de recursos de la agroindustria como oportunidad para dar valor a los residuos orgánicos no aprovechados. En este escenario, el proyecto marco que da lugar a este artículo explora, a través de estrategias de ecodiseño, economía circular y diseño guiado por el material (Material Driven Design), las oportunidades de creación y aplicación de materiales biocompuestos, donde el diseño como actividad profesional se visualiza como una estrategia competitiva para el aprovechamiento de 
residuos agroindustriales y el cierre de brechas tecnológicas en aplicaciones productivas de mediana escala. Este artículo de investigación presenta entonces, un análisis comparativo cualitativo de tres casos de estudio, que permite evidenciar cómo la integración de esa mirada entre lo técnico y lo experiencial realza las potencialidades de aplicación y transformación en contexto de los materiales aquí expuestos, que lograron una estabilidad material suficiente para su transformación y uso, su propio balance entre lo sensorial y estético, de bajo impacto ambiental, y con procesos de conformación y producción viables y escalables basados en procesos low tech de fácil transferencia tecnológica.

\section{Abstract:}

The circular economy implementation policies in Colombia have prioritized the use of agribusiness resources as an opportunity to give value to unused organic waste. In this scenario, the framework project that gives rise to this article explores, through ecodesign strategies, circular economy and Material Driven Design, the opportunities for creating and applying biocomposite materials, where design as professional activity is seen as a competitive strategy for the use of agro-industrial waste and allows closure of technological gaps in medium-scale productive applications. This research article presents then, a qualitative comparative analysis of three cases, which allows to show how the integration of this gaze between the technical and the experiential enhances the potentialities of application and transformation in context of the materials presented here, which achieved: sufficient stability for its conformation and use, its own balance between sensory and aesthetic, low environmental impact, and the definition of low tech based processes of easy technology transfer, viable and scalable. 


\section{INTRODUCCIÓN}

La economía circular por definición se constituye como una estrategia reparadora y regenerativa, la cual podemos comprender cómo el modelo que procura mantener el valor de los recursos que ya están en circulación en el mundo en todo momento, reduciendo así, la demanda de recursos nuevos gracias a que distingue entre ciclos técnicos y biológicos para la regeneración de los mismos (Ellen Macarthur Foundation, S.F.). Ésta prioriza el diseño como estrategia para que los recursos regresen a la producción, los productos desarrollados sean de calidad y duraderos, que estos se puedan deconstruir para su reparación o aprovechamiento, y que generen ciclos cerrados (regenerativos) que sean beneficiosos para la construcción de capital natural (Ellen Macarthur Foundation, S.F.).

En Colombia, como se explica en la Guía Empresarial de Economía circular (Colombia Productiva 2020), la agroindustria genera 65\% residuos orgánicos no aprovechados. Considerando que la producción agrícola en el mundo es responsable del 30\% del consumo total de energía y un 22\% del total de las emisiones de gases de efecto invernadero (Organización de Naciones Unidas [ONU], 2015), se puede afirmar que la agricultura es una de las actividades humanas de mayor impacto ambiental, tanto en la producción, como en la disposición de residuos. 
Con base en esto, la Estrategia Nacional de Economía Circular (Gobierno de la República de Colombia, 2019) y el Departamento Nacional de Planeación [DNP] (2018) prioriza el aprovechamiento de residuos agroindustriales, con un potencial de generar valor agregado en un $25,61 \%$. Además, expone que “( ...) los biomateriales (procedentes de la actividad transformadora de productos agroforestales) constituyen nichos de oportunidad para mejorar la eficiencia de recursos (...) y crear actividades emergentes de bioeconomía." (DNP, 2018:13). Lo anterior, se profundiza en el creciente interés de las empresas por desarrollar productos y estrategias que permitan la sostenibilidad en los ámbitos ambiental, social y económico, y al mismo tiempo, ser competitivos en el mercado.

Este planteamiento abre la oportunidad de explorar el diseño y aplicación de materiales alternativos y sostenibles a partir de los residuos generados en la agroindustria. Ahora bien, aún así, uno de los grandes retos que se establece en el desarrollo de estos materiales y productos es la brecha tecnológica que existe entre los laboratorios y los escenarios de transformación (Centro de Materiales, 2019), ya que los procesos para generar, por ejemplo, biopolímeros a partir de la síntesis de almidón suceden en escenarios industriales y laboratorios de mayor complejidad. Es precisamente allí, donde se encuentra una oportunidad de abordar procesos de apropiación social de CTeI enfocados en procesos de co-creación y desarrollo de alternativas productivas de baja complejidad tecnológica en las que el diseño puede ser un factor relevante tanto a nivel productivo como en su capacidad de llevar esos resultados a mercados viables en desarrollo de producto.

Por su parte, la exploración de materiales en el diseño está relacionada con la comprensión de diversas dimensiones, como lo indican Michael Ashby y Kara Johnson (2014), lo que es importante hoy en día es el proceso de encontrar soluciones que sean significativas para las personas, que detonen nuevas experiencias y que sean sostenibles, por tanto, la elección del material no se limita a un factor puramente técnico y de eficiencia, ni es una elección estilística, sino que se trata de una combinación entre ciencia y arte. 
En relación a esto, desde el diseño se han desarrollado metodologías que facilitan la intervención del material mismo como parte del quehacer creativo, aparecen tendencias impulsadas por la democratización de las tecnologías y la creciente tendencia a la personalización de los productos -y materiales-, que han llevado a que los diseñadores y creadores intervengan su propio material como parte de su proceso creativo (Ayala y Rognoli, 2015), generando sus propias modificaciones y características para tener diversos resultados, a través de metodologías Do It Yourself (DIY), es decir, cocinando materiales en su propia casa. El diseñador a través de estas metodologías de exploración y comprensión de las propiedades funcionales, estéticas y de transformación de estos materiales está en capacidad de generar propuestas donde se valoran dichas propiedades.

Es así, que el diseño como actividad profesional se visualiza como una estrategia competitiva que se propone hacer un puente entre la complejidad técnica del laboratorio y los procesos de exploración y producción en casa de los materiales, reduciendo la brecha tecnológica y facilitando el aprovechamiento de los recursos mencionados, en escalas pequeñas y medianas de baja complejidad tecnológica, con aplicaciones en diseño de producto que consideran el material desde la estética, la experiencia a través de los sentidos, su uso y su transformación. En ese sentido, los materiales biocompuestos, conformados por una matriz (resina) y una base de refuerzo (fibra, carga mineral, etc) son potenciales para su aprovechamiento (Ramos Riascos \& Caro Vives, 2014).

Este artículo, tiene por objetivo presentar un análisis basado en tres experiencias de experimentación y aplicación con materiales biocompuestos a partir de fuentes recuperadas resultado de dichas metodologías, en donde, a través de un análisis de sus propiedades desde la mirada del diseño, pueden ser aprovechados en sus aspectos funcionales, estéticos y posibilidades de transformación, con los que es posible generar propuestas de materiales y productos que se conectan con diferentes contextos y cadenas productivas, con un nivel interesante de viabilidad como alternativa productiva y de diseño, y con buen potencial de escalabilidad. 


\section{METODOLOGÍA}

Para el desarrollo del análisis aquí presentado se estudian tres casos tomados del escenario de Proyecto de Grado en la línea de Ecodiseño y Sostenibilidad [PG-Eco] del programa de Diseño Industrial de la Universidad El Bosque, que ha venido trabajando desde hace varios años en la experimentación de mezclas de diferentes fibras naturales provenientes de residuos de producción agroindustrial mezclados con resinas naturales, biopolímeros y resinas de base acuosa, con el fin de desarrollar materiales y aplicaciones de fácil degradación y mínimo impacto ambiental.

El proceso de desarrollo de dichos casos consiste en una combinación de metodologías como Material Driven Design Method (Karana, et.al., 2015) que establece la exploración técnica y de la experiencia del usuario; métodos de Circular Design Guide (Ellen Macarthur Foundation, IDEO, 2016) para entender el desarrollo de nuevas propuestas formales como producto competitivo, valioso y en evolución, y principios de DIY Materials (Ayala, Rognoli, 2015) como referente de experimentación y registro. Así mismo, se orientan desde principios de economía circular y diseño sistémico (Bistagnino, 2009) donde las salidas de un sistema son alimento para otro con base en las relaciones entre los actores.

El proceso empieza por una exploración de combinaciones de residuos de agroindustria con aglutinantes biodegradables de bajo impacto ambiental. De esta experimentación se definen características técnicas (cualitativo), aspectos estéticos y sensoriales, y procesos de transformación con tecnologías de baja complejidad. Luego, se priorizan las propiedades según su valor agregado, y se conecta con mercados para los que se propone el diseño de producto. Paralelamente, se diseña el ciclo de vida que da forma a la cadena de valor.

Los casos aquí presentados fueron elegidos como relevantes para el análisis, porque tienen en común una fuente recuperada de agroindustria y dieron como resultado una aplicación interesante y viable en un contexto que valora sus propiedades estéticas, técnicas y productivas, con bajo impacto ambiental. Los tres fueron propuestos para el desarrollo de empaques relacionados con alimentos, como respuesta al consumo de plásticos de un solo uso y al reto de aprovechar su capacidad de ser biodegradados rápidamente sin contaminar su contenido. La mayor diferencia es que provienen de escenarios geográficos y productivos muy diferentes, pero que tienen un alto potencial de impacto local. 
Para el presente análisis comparativo se revisan de manera cualitativa los siguientes aspectos:

\section{Aspectos Técnicos}

Se refiere a aquellas propiedades físicas y químicas que, vistas desde el diseño, se valoran en desempeños y limitaciones del material (Karana et.al. 2015) para ser aplicado en productos, tales como su resistencia a la tensión y al rasgado, flexibilidad, dureza, elasticidad, resiliencia, resistencia a agentes químicos y biológicos (biodegradación), resistencia a la humedad y temperatura.

\section{Aspectos estéticos y de experiencia de material}

Se refiere a los aspectos que se pueden percibir en el material, y cómo son interpretados por las personas desde su subjetividad. De una parte, se analizan los elementos visuales (Wong, 2014): color, textura, forma, tamaño, etc. que hacen parte de la interpretación plástica y construcción de una estética particular. Por otro lado, se indican los niveles de experiencia como sugerido en Material Driven Design Method. (Karana, et.al. 2015, p. 41): nivel sensorial (sentidos), interpretativo (significados), afectivo (emociones) y performativo (funcionalidades y acciones).

\section{Aspectos Productivos}

Se refiere a las posibilidades de transformación que puede tener el material y que lo conectan con contextos productivos ya existentes. Aquí, se consideran tanto los procesos, como la cadena de valor que podría asociarse con el material. Se indican procesos de baja complejidad técnica tales como moldeado con calor y presión, corte, costura, confección, plegado y pegado, ensamble simple, y relación con otros materiales.

\section{Aspectos ambientales}

Permite comprender las implicaciones ambientales desde una perspectiva holística a través del análisis de ciclo de vida cualitativo y el diseño sistémico. Son analizados el nivel de aprovechamiento (uso de residuos y recursos renovables), la viabilidad e impacto en contexto, mínimo desperdicio en todas las fases del ciclo de vida, los impactos sobre la salud humana y ecosistemas, y la calidad y tipo de disposición final que se plantea. Vale la pena señalar que como parte de la metodología de PG-Eco se diseña pensando en que los recursos provengan de fuentes recuperadas y se conviertan en nutrientes para otros sistemas técnicos o biológicos (Mcdonough, W., \& Braungart, M., 2005) y se aplican diferentes estrategias de ecodiseño para garantizar el mínimo impacto ambiental. 
Se presentan como evidencia tres casos de estudio provenientes de la línea de proyectos de grado de diseño industrial con énfasis en ecodiseño y sostenibilidad:

- "Ambar: el amor tiene forma de mango" (Merchán, 2019) Diseño de snacks, empaque y salsas a partir de la sobreproducción de mango en Ciénaga, Magdalena. Material: cuero de mango, mango deshidratado, pulpa de mango (Figura 1).

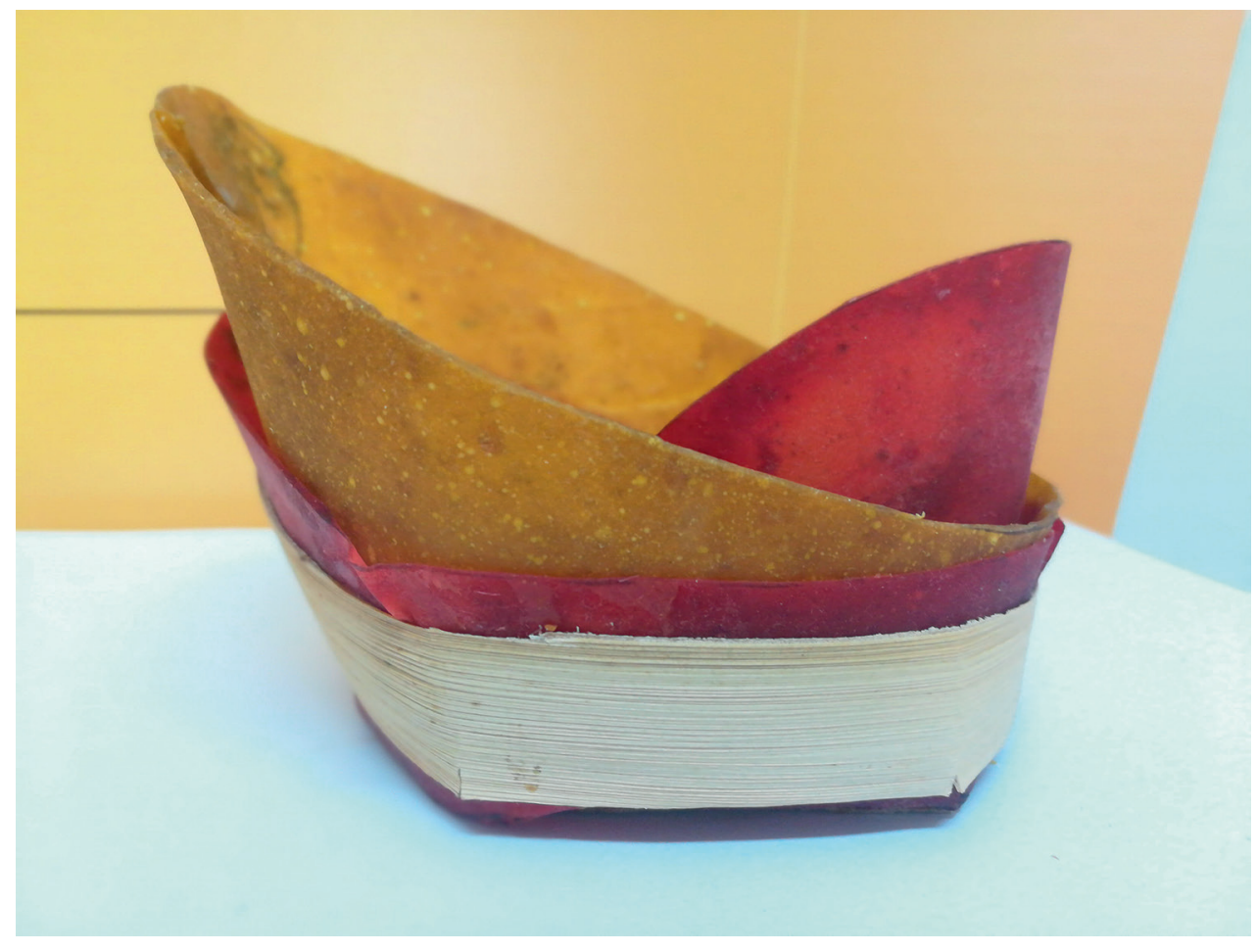

Figura 1. "AMBAR: el amor tiene forma de mango" Cuero de Mango. Entregas finales de proyectos de grado de diseño industrial semestre 2019-1.

Fuente: autora. 
- Egg Last: sistema de empaque biodegradable para comida B.A.R.F (Mateus, 2019) Material a base de cáscara de huevo y aglutinante natural, empaque comestible a partir de residuos de la misma producción de comida B.A.R.F. (Figura 2).

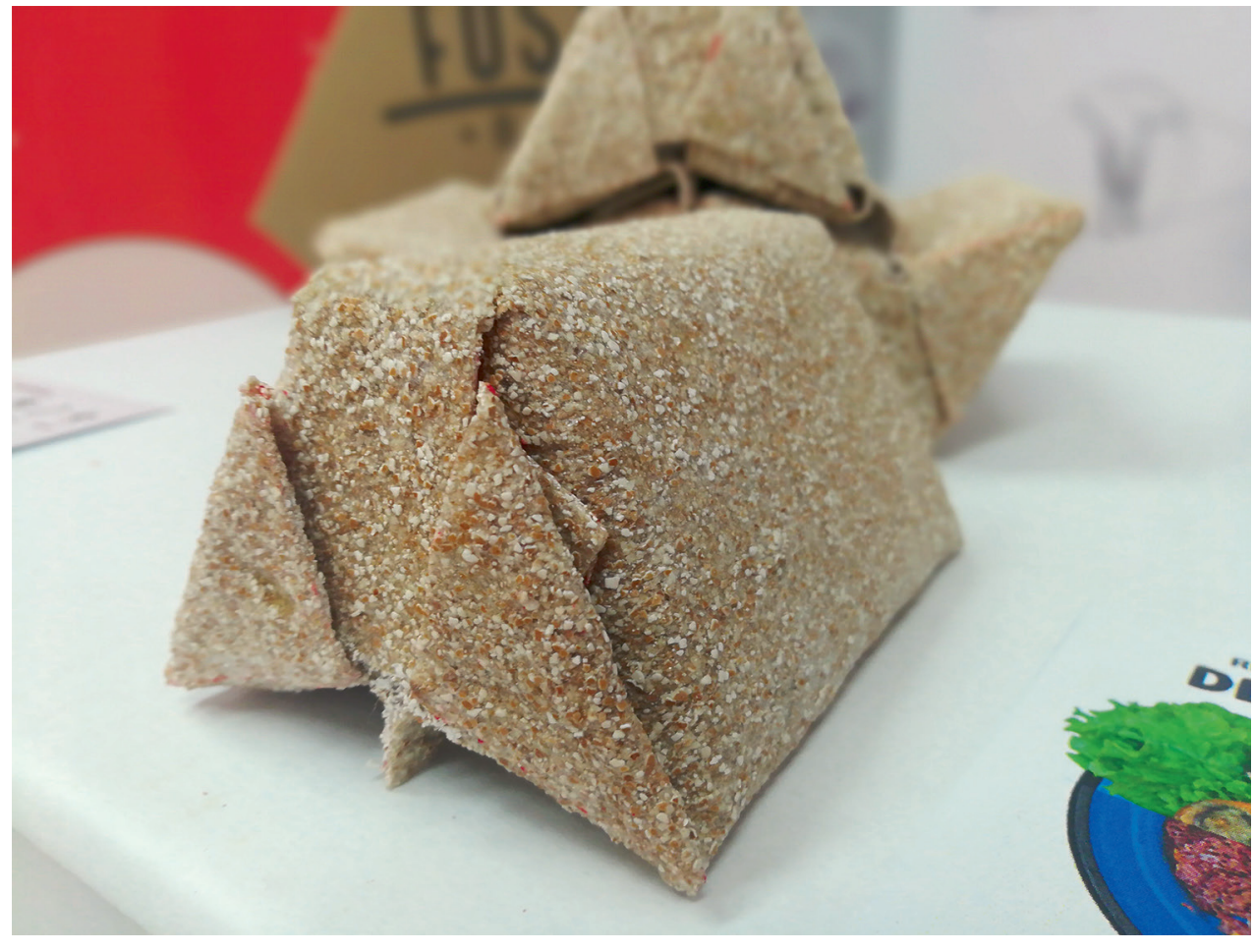

Figura 2. Egg Last: Sistema de empaque biodegradable para comida B.A.R.F. Entregas finales de proyectos de grado de diseño industrial semestre 2019-1. 
- Suamne: valorización del bagazo de caña panelera como material alternativo para el diseño y desarrollo de productos aglomerados biodegradables (Nuncira, 2018) (Figura 3). Empaques y menaje biodegradables y germinables a base de bagazo de caña panelera.

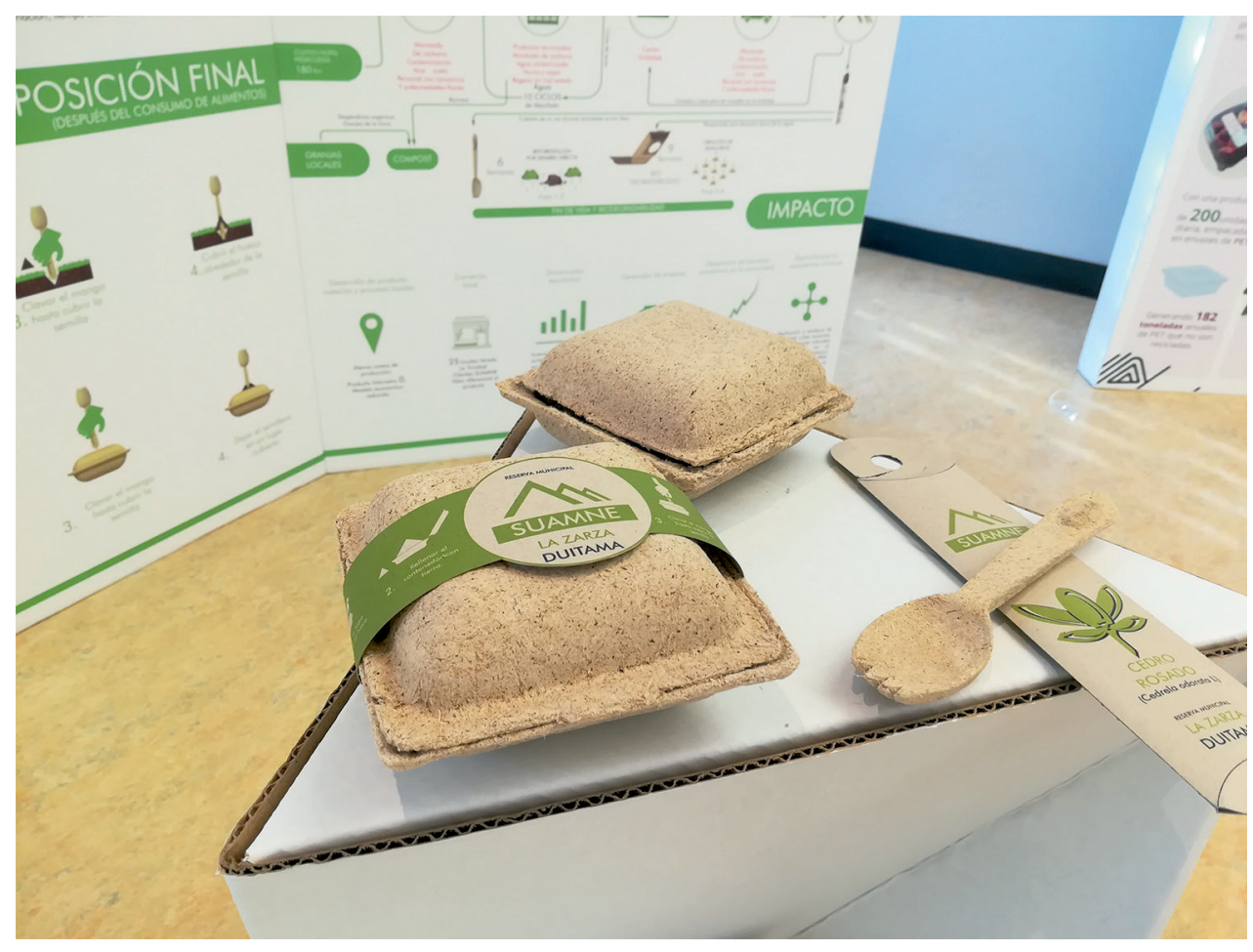

Figura 3. Suamne: Valorización del bagazo de caña panelera como material alternativo para el diseño y desarrollo de productos aglomerados biodegradables.

Entregas finales de proyectos de grado de diseño industrial semestre 2018-1.

Fuente: autora.

\section{RESULTADOS}

Al analizar estos casos, se puede evidenciar el aprovechamiento del residuo agroindustrial, el desarrollo de procesos para su conformación y transformación, y las propiedades formales, estéticas y experienciales que se lograron en su exploración.

Como factores diferenciales, se descubrió que los materiales cuya matriz contiene geles o gelatina son más flexibles y se trabajan mejor como lámina, y los que tienen refuerzo con fibra son más resistentes a esfuerzos mecánicos y se conforman mejor por moldeo porque son más estables. Cada material presentó sus propias caracte- 
rísticas sensoriales que superan los aspectos visuales, donde se resalta el color, translucidez y aroma en el cuero de mango, la textura en la fibra de caña panelera, y la geometría que se logra con la película de cáscara de huevo, abriendo posibilidades a aplicaciones diversas y competitivas.

Como similitudes cabe resaltar que la conformación de los tres se logró a través de procesos de baja y mediana complejidad con los recursos encontrados en contexto, y su manipulación se realiza con medios tradicionales como el moldeo con vapor y el troquelado, lo que facilitaría su apropiación y transferencia tecnológica, así como la inclusión de capacidad instalada para la transformación de otros materiales tradicionales.

Por su parte, los tres materiales y sus aplicaciones para empaques contribuyen al cumplimiento de los principios de economía circular (Ellen Macarthur Foundation, S.F.): Principio 1: diseñar los residuos y la contaminación: El origen de los recursos viene del descarte de otras industrias, reduciendo así el desperdicio de los mismos. De otro lado, los productos y los materiales aquí descritos están inscritos en ciclos biológicos, conectando el residuo orgánico con un uso que luego llegará de nuevo a la tierra; Principio 2: Mantener los productos y servicios en uso: En este caso, aunque los productos son de corta durabilidad, los materiales son valorizados y provienen del aprovechamiento de un residuo, alargando la vida útil del recurso, y también se integran en procesos que facilitan su aprovechamiento en cadenas de valor colaborativas que generan nuevas conexiones en simbiosis industrial; Principio 3: Regenerar los sistemas naturales: estos materiales son de origen biológico y al ser mezclados con el contenido de las propuestas realizadas (carnes, comida, snacks), se enriquecen y facilitan el proceso de compostaje.

\section{Aspectos técnicos}

En cuanto a los aspectos técnicos, como se muestra en la tabla 1, se pueden encontrar limitaciones en la especificación de sus características, ya que carece de pruebas técnicas. Sus pruebas fueron de tipo empírico y comparativo, para cada material se estableció un protocolo de prueba al que fueron sometidas diferentes probetas de cada material. Sin embargo, se logra llegar a una estabilidad de material suficiente para caracterizarlo y aplicarlo a diseño de productos, donde se resaltan las propiedades mecánicas como elasticidad, impermeabilidad y resistencia a la tensión y compresión, así como no toxicidad y resistencia a agentes biológicos, lo que resulta en una durabilidad suficiente para contener y proteger alimentos en corto plazo. 
El de fibra de caña tuvo un proceso de experimentación que buscó definir la mejor combinación de aglutinante - material y el proceso más sencillo con resultados más estables. Realizó pruebas de espesor de material en proceso de producción, consistencia, luminosidad y presencia de hollín al ser quemado, precisión de detalle en moldeo, impermeabilidad y sensibilidad a la temperatura, definiendo que el proceso de prensado con calor mejora la resistencia pero disminuye la impermeabilidad (Nuncira Rincón, Buraglia Osorio, Gómez Puertas, 2018) Así se elige realizar láminas de bagazo sin aglutinante en un proceso de prensado, y posterior acabado con cera para mantener la impermeabilidad.

El proceso del cuero de mango se concentró en lograr la mezcla que mantuviera las propiedades durante más tiempo y de manera estable, comparando diferentes mezclas hasta definir la que dio mayor estabilidad y flexibilidad para su manipulación (pruebas de resistencia al peso y a la humedad), y con especial atención en no perder las propiedades organolépticas como el aroma (Merchán Camelo, Buraglia Osorio, 2019).

Por último, las pruebas de la lámina de cáscara de huevo se realizaron a partir de las propiedades requeridas para el empaque al que se quería llegar, es decir, se buscó la mejor combinación de ingredientes permitieran soportar el peso y humedad, que se pudiera conformar como lámina y transformación por plegados, resistencia al rasgado y resistencia a temperatura de congelación. Así mismo, las pruebas de asepsia se basaron en investigación y en las condiciones de fabricación (Mateus Guerra, Buraglia Osorio, 2019). 


\begin{tabular}{|c|c|c|c|}
\hline Descripción & $\begin{array}{l}\text { Cuero de mango } \\
\text { Laminado }\end{array}$ & $\begin{array}{l}\text { Pulpa de caña panelera } \\
\text { prensada con acabado } \\
\text { impermeable }\end{array}$ & $\begin{array}{l}\text { Lámina de cáscara de } \\
\text { huevo }\end{array}$ \\
\hline- & $\begin{array}{l}\text { lpa de mango de agua } \\
\text { gelatina }\end{array}$ & $\begin{array}{l}\text { gazo de caña panelera } \\
\text { era de abejas. }\end{array}$ & $\begin{array}{l}\text { le huevo } \\
\text { gelatina }\end{array}$ \\
\hline 11 & $\begin{array}{l}\text { Resistencia mecánica a la } \\
\text { tensión } \\
\text { Impermeable } \\
\text { Elástico } \\
\text { Flexible } \\
\text { Estable al corte } \\
\text { Maleable a temperatura } \\
\text { ambiente } \\
\text { Resistente a baja } \\
\text { temperatura }\end{array}$ & $\begin{array}{l}\text { Resistente a la } \\
\text { compresión y a rasgado. } \\
\text { Estabilidad estructural } \\
\text { Impermeable (acabado) } \\
\text { Liviano }\end{array}$ & $\begin{array}{l}\text { Resistencia al rasgado } \\
\text { y estabilidad a } \\
\text { compresión } \\
\text { Impermeable } \\
\text { Maleable a } \\
\text { temperatura ambiente } \\
\text { Resistente a bajas } \\
\text { temperaturas (se } \\
\text { congela) } \\
\text { Liviano }\end{array}$ \\
\hline $\begin{array}{l}\text { Propiedades } \\
\text { químicas }\end{array}$ & $\begin{array}{l}\text { No tóxico } \\
\text { Resistente a agentes } \\
\text { ambientales } \\
\text { Biodegradable (sin } \\
\text { refrigeración) }\end{array}$ & $\begin{array}{l}\text { No tóxico } \\
\text { Alto potencial energético } \\
\text { Biodegradable } \\
\text { (dispuesto en medio } \\
\text { ambiente) }\end{array}$ & $\begin{array}{l}\text { No tóxico } \\
\text { Resistente a agentes } \\
\text { biológicos (contenido) } \\
\text { Biodegradable y } \\
\text { comestible (perro) }\end{array}$ \\
\hline $\begin{array}{l}\text { Desempeño y } \\
\text { aplicación }\end{array}$ & $\begin{array}{l}\text { Desarrollado para } \\
\text { empaques de alimentos } \\
\text { para llevar, no para } \\
\text { conservar. Utensilios de } \\
\text { único uso. Porta salsas y } \\
\text { chips. }\end{array}$ & $\begin{array}{l}\text { Platos y cubiertos } \\
\text { germinables, portátiles } \\
\text { para escenarios de } \\
\text { campamento y trekking. } \\
\text { Se usa para iniciar } \\
\text { fogatas. }\end{array}$ & $\begin{array}{l}\text { Empaque de unidades } \\
\text { de alimento para perro } \\
\text { tipo B.A.R.F. para } \\
\text { su almacenamiento } \\
\text { y distribución. Sin } \\
\text { desperdicio. }\end{array}$ \\
\hline
\end{tabular}

Tabla 1. Comparación de aspectos técnicos.

Fuente: autora.

\section{Aspectos estéticos y experienciales}

En cuanto a los aspectos estéticos y experienciales (Tabla 2) se puede evidenciar que, respecto al proceso de exploración desde la mirada del diseñador se establece un balance entre los aspectos sensoriales y técnicos, donde se configura una estética propia del diseño con materiales alternativos en la que la textura y el color son 
característicos y recuerdan el pasado orgánico del material. Así mismo, su conformación como producto reclama el desarrollo de geometrías básicas que sea posible reproducir con la menor cantidad de procesos de transformación y a partir de conformaciones del material que permitan su manipulación, por ejemplo, laminados para plegado, o viscosos para moldeo.

\begin{tabular}{|c|c|c|c|}
\hline Descripción & $\begin{array}{l}\text { Cuero de mango } \\
\text { Laminado }\end{array}$ & $\begin{array}{l}\text { Pulpa de caña } \\
\text { panelera prensada } \\
\text { con acabado } \\
\text { impermeable }\end{array}$ & $\begin{array}{l}\text { Lámina de cáscara de } \\
\text { huevo }\end{array}$ \\
\hline $\begin{array}{l}\text { Elementos } \\
\text { formales }\end{array}$ & $\begin{array}{l}\text { Diversidad de colores } \\
\text { intensos en tonos } \\
\text { cálidos } \\
\text { Textura lisa } \\
\text { Acabado brillante } \\
\text { Translúcido } \\
\text { Composición en formas } \\
\text { lineales y geometría } \\
\text { básica (estabilidad del } \\
\text { corte) }\end{array}$ & $\begin{array}{l}\text { Color neutro } \\
\text { de la fibra, baja } \\
\text { saturación } \\
\text { Textura fibrosa } \\
\text { irregular } \\
\text { Acabado mate } \\
\text { Formas } \\
\text { redondeadas } \\
\text { tridimensionales. } \\
\text { Geometría regular. }\end{array}$ & $\begin{array}{l}\text { Color texturado blanco } \\
\text { y rojizo baja saturación } \\
\text { Textura punteada fina, } \\
\text { Acabado mate } \\
\text { Diseño plegado de } \\
\text { formas triangulares } \\
\text { inspirado en origami y } \\
\text { arquitectura de papel. }\end{array}$ \\
\hline Nivel sensorial & $\begin{array}{l}\text { Textura suave y elástica } \\
\text { al tacto } \\
\text { Aroma de la fruta. } \\
\text { Flexible y pesado }\end{array}$ & $\begin{array}{l}\text { Liviano, textura } \\
\text { rugosa al tacto, seco, } \\
\text { sin aroma, rígido. } \\
\text { Liviano }\end{array}$ & $\begin{array}{l}\text { Textura rugosa al tacto, } \\
\text { temperatura fría, sin } \\
\text { aroma, flexible, blando. }\end{array}$ \\
\hline $\begin{array}{l}\text { Nivel } \\
\text { interpretativo }\end{array}$ & $\begin{array}{l}\text { Tropical, exótico, } \\
\text { colorido }\end{array}$ & $\begin{array}{l}\text { Natural, desechable, } \\
\text { ecológico }\end{array}$ & Orgánico, frío \\
\hline Nivel afectivo & Curioso, experimental & $\begin{array}{l}\text { Responsable, } \\
\text { ecológico, natural }\end{array}$ & Neutro \\
\hline $\begin{array}{l}\text { Nivel } \\
\text { performativo }\end{array}$ & $\begin{array}{l}\text { corta vida útil, } \\
\text { enriquece la experiencia } \\
\text { de comer }\end{array}$ & $\begin{array}{l}\text { liviano, germinable, } \\
\text { biodegradable }\end{array}$ & $\begin{array}{l}\text { Todo se come, sin } \\
\text { desperdicio }\end{array}$ \\
\hline
\end{tabular}




\section{Aspectos productivos}

Los procesos propuestos (Tabla 3) se definen a partir de revisar procesos locales y de baja complejidad posibles en pequeña escala y con técnicas DIY replicables. Para esto, es importante llevar un registro organizado de resultados con el fin de facilitar su reproducción en otros escenarios, y con la intención de aplicar low tech que sea muy fácil de replicar y apropiar con elementos que ya existen o que se pueden configurar en contexto, tales como bastidores (mango), compresión y moldeo de pulpa (caña panelera) y mezcla, laminado y plegado (cáscara de huevo). Esto da como resultado producto viable, contextualizado y para mercados definidos.

\begin{tabular}{|c|c|c|c|}
\hline Descripción & $\begin{array}{l}\text { Cuero de mango } \\
\text { Laminado }\end{array}$ & $\begin{array}{l}\text { Pulpa de caña panelera } \\
\text { prensada con acabado } \\
\text { impermeable }\end{array}$ & $\begin{array}{l}\text { Lámina de cáscara de } \\
\text { huevo }\end{array}$ \\
\hline Presentación & Lámina & Pulpa moldeable & Lámina \\
\hline Conformación & $\begin{array}{l}\text { Licuado, mezcla } \\
\text { y secado al sol en } \\
\text { bastidor }\end{array}$ & $\begin{array}{l}\text { Triturado, mezcla con } \\
\text { cera de abejas derretida, }\end{array}$ & $\begin{array}{l}\text { Triturado, mezcla } \\
\text { con aglutinante y } \\
\text { refrigeración }\end{array}$ \\
\hline Transformación & $\begin{array}{l}\text { Corte a mano o } \\
\text { troquelado y plegado }\end{array}$ & $\begin{array}{l}\text { Prensado de pulpa con } \\
\text { vapor y troquelado }\end{array}$ & $\begin{array}{l}\text { Corte, pliegue y } \\
\text { congelación }\end{array}$ \\
\hline Corte & $\begin{array}{l}\text { Troquelado o corte a } \\
\text { mano }\end{array}$ & Troquelado & Corte a mano \\
\hline Pegado & $\begin{array}{l}\text { Con la misma fórmula } \\
\text { líquida }\end{array}$ & $\begin{array}{l}\text { Adhesivos con base } \\
\text { acuosa o con cera de } \\
\text { abeja }\end{array}$ & Con el aglutinante \\
\hline $\begin{array}{l}\text { Contexto } \\
\text { y cadena } \\
\text { productiva }\end{array}$ & $\begin{array}{l}\text { Se puede trabajar } \\
\text { en las condiciones } \\
\text { ambientales locales } \\
\text { (Ciénaga, Magdalena) } \\
\text { donde el ambiente es } \\
\text { cálido y seco, ideal } \\
\text { para el secado del } \\
\text { material. } \\
\text { El producto se proyectó } \\
\text { para escenarios } \\
\text { turísticos de la costa } \\
\text { Atlántica. }\end{array}$ & $\begin{array}{l}\text { Los recursos y su } \\
\text { fabricación se ubicaron } \\
\text { en la zona panelera de } \\
\text { Santander y Boyacá, y } \\
\text { el producto se proyectó } \\
\text { para implementarlo } \\
\text { en un parque natural } \\
\text { de la región como } \\
\text { estrategia para eliminar } \\
\text { los plásticos de un sólo } \\
\text { uso y como germinable } \\
\text { para dispersar semillas } \\
\text { nativas. }\end{array}$ & $\begin{array}{l}\text { El recurso base } \\
\text { (cáscara de huevo) } \\
\text { es un subproducto } \\
\text { de la producción } \\
\text { del alimento } \\
\text { B.A.R.F. en una } \\
\text { empresa de Tunja. } \\
\text { Se puede conformar } \\
\text { y transformar en } \\
\text { la misma planta } \\
\text { y aprovechar la } \\
\text { infraestructura de } \\
\text { refrigeración. }\end{array}$ \\
\hline
\end{tabular}




\section{Aspectos ambientales}

En cuanto a la conformación de los materiales, se logra una proporción de por lo menos $70 \%$ de materia recuperada, con una durabilidad corta, haciendo que su aplicación tenga la condición de ser desechados rápidamente, y transformados a partir de procesos locales, lo que facilita el cierre de ciclos y aprovechamiento de los recursos, en coherencia con principios de economía circular (Tabla 4). Estos procesos están conectados con el contexto local, un mercado y una cadena productiva de baja complejidad, lo que facilita su transferencia y escalamiento.

\begin{tabular}{|c|c|c|c|}
\hline & Ambar & Suamne & EggLast \\
\hline Descripción & $\begin{array}{l}\text { Cuero de mango } \\
\text { Laminado }\end{array}$ & $\begin{array}{l}\text { Pulpa de caña panelera } \\
\text { prensada con acabado } \\
\text { impermeable }\end{array}$ & $\begin{array}{l}\text { Lámina de cáscara de } \\
\text { huevo }\end{array}$ \\
\hline $\begin{array}{l}\text { Problema } \\
\text { inicial }\end{array}$ & $\begin{array}{l}\text { Sobreproducción de } \\
\text { mango en Magdalena: } \\
\text { pérdida de } 4800 \\
\text { toneladas al año. }\end{array}$ & $\begin{array}{l}\text { Desperdicio de bagazo } \\
\text { de caña en contexto de } \\
\text { cultivo aprox. } 540.000 \\
\text { toneladas al año. }\end{array}$ & $\begin{array}{l}\text { Desperdicio de bolsas de } \\
\text { PEBD } 100 \mathrm{Kg} \text { aprox. al } \\
\text { año, contaminado con } \\
\text { residuos de carne. }\end{array}$ \\
\hline $\begin{array}{l}\text { Recurso } \\
\text { recuperado }\end{array}$ & $\begin{array}{l}\text { Composición } 70 \% \text { Pulpa } \\
\text { de mango }\end{array}$ & $\begin{array}{l}\text { Composición } 60 \% \text { bagazo } \\
\text { de caña }\end{array}$ & $\begin{array}{l}\text { Composición } 20 \% \text { cáscara } \\
\text { de huevo recuperada }\end{array}$ \\
\hline $\begin{array}{l}\text { Tiempo y } \\
\text { condiciones de } \\
\text { biodegradación }\end{array}$ & $\begin{array}{l}\text { Conservado: } 1 \text { año o más } \\
\text { En ambiente: } 1 \text { a } 3 \text { meses }\end{array}$ & $\begin{array}{l}\text { Enterrado o a intemperie: } \\
3 \text { a } 4 \text { meses }\end{array}$ & $\begin{array}{l}\text { Húmedo: } 1 \text { mes a la } \\
\text { intemperie } \\
\text { Seco: } 3 \text { meses a la } \\
\text { intemperie }\end{array}$ \\
\hline Ecotoxicidad & No tóxico - comestible & $\begin{array}{l}\text { No tóxico. Si incinerado, } \\
\text { genera CO2 }\end{array}$ & No tóxico - comestible \\
\hline $\begin{array}{l}\text { Consumo de } \\
\text { energía }\end{array}$ & $\begin{array}{l}\text { Energía solar (secado), } \\
\text { corte y armado (puede } \\
\text { ser manual) }\end{array}$ & $\begin{array}{l}\text { Maquinado y prensado } \\
\text { con vapor (calor y } \\
\text { movimiento) }\end{array}$ & $\begin{array}{l}\text { Refrigeración para } \\
\text { conformación y } \\
\text { conservación (ya hace } \\
\text { parte de cadena de valor) }\end{array}$ \\
\hline
\end{tabular}




\begin{tabular}{|c|c|c|c|}
\hline $\begin{array}{l}\text { Ciclo de vida y } \\
\text { estrategias de } \\
\text { ecodiseño }\end{array}$ & $\begin{array}{l}\text { Producto local } \\
\text { Recursos recuperados de } \\
\text { agroindustria } \\
\text { Mínimo consumo } \\
\text { energético en procesos } \\
\text { Monomaterial } \\
\text { Corta durabilidad } \\
\text { Biodegradable }\end{array}$ & $\begin{array}{l}\text { Producto local } \\
\text { Recurso recuperado de } \\
\text { agroindustria } \\
\text { Mínimo consumo } \\
\text { energético } \\
\text { Diseño regenerativo } \\
\text { (germinable) } \\
\text { Corta durabilidad } \\
\text { Aprovechamiento } \\
\text { energético }\end{array}$ & $\begin{array}{l}\text { Producto local dentro de } \\
\text { su cadena de valor. } \\
\text { Recurso recuperado de } \\
\text { agroindustria. } \\
\text { Comestible (zero waste). } \\
\text { Monomaterial } \\
\text { corta durabilidad. }\end{array}$ \\
\hline
\end{tabular}

Tabla 4. Comparación de aspectos ambientales.

Fuente: autora.

En cuanto a la apropiación y transferencia, el proceso de co-creación que involucra a los actores locales donde se produce el residuo facilita la comprensión e integración de procesos existentes, que, con mínimas modificaciones, pueden adaptarse para la conformación y transformación de los materiales propuestos. 


\section{CONCLUSIONES Y RECOMENDACIONES}

El proceso de diseño a partir de los materiales permite al diseñador una comprensión integral de sus características más allá de los aspectos técnicos y así, proyectar la experiencia en la plástica, integrando las posibilidades de transformación y configuración que da el material a las aplicaciones como fundamento para las decisiones en la composición, que además da cuenta de su origen orgánico y conecta al usuario con él.

La experimentación en el proceso es la base que permite comprender estas dimensiones, pero requiere de un registro del proceso suficientemente riguroso que permita articular las mejores características de la forma, la funcionalidad y la viabilidad en contexto. Debe además, trascender a escenarios de diseño de producto con un mercado definido y unos procesos escalables que permitan acabados de calidad y resaltar los aspectos sensoriales como valor en la forma.

Por otro lado, existen en efecto múltiples oportunidades en la exploración de materiales recuperados, ya que su uso reduciría el impacto tanto de los residuos generados, como de los materiales que pueden reemplazar y que hoy en día representan un riesgo para los ecosistemas, pero se proyecta diseñar para la durabilidad, de manera que lo que es de corto uso, se degrade igualmente rápido y fácil.

Una dificultad que presentan estos casos se relaciona con los costos comparando procesos tradicionales con los alternativos, ya que, por el momento, los procesos son artesanales e implican más tiempo, incrementando la inversión de recursos humanos y económicos, pero se requiere entonces continuar en la búsqueda de simplificación y escalamiento de los procesos y conectar los actores que servirían como proveedores de los recursos. Sin embargo, se ha evidenciado que sí existen mercados interesados en productos hechos con materiales alternativos, considerando una creciente importancia por los aspectos ambientales y por el futuro del planeta para los consumidores, así como la evolución en las políticas que reclaman cambios en la industria.

Por último, el diseño actúa como articulador entre el mercado, las propiedades del material, y la viabilidad, desarrollando productos útiles, que valorizan las propiedades a través de la forma y la función, y facilita la transición a procesos contextualizados. Desde el ámbito académico, como parte de la formación de los 
diseñadores es relevante fomentar la exploración de los materiales que provienen de fuentes alternativas a través de procesos de investigación-creación (Ballesteros y Beltrán, 2018) que permitan encontrar no sólo las propiedades sino también las aplicaciones en escenarios productivos y comerciales. Desde el ámbito institucional promover la generación de normativas y políticas públicas que faciliten la comprobación de los materiales de manera que sea más fácil su aplicación en industria. Por su parte en el ámbito empresarial, el diseñador estará en capacidad de encontrar valor en la abundancia, reconociendo las oportunidades que hay en la recuperación de recursos a través del análisis y reconocimiento de las propiedades de los materiales que le permiten proponer aplicaciones alternativas e innovadoras, que además van en sintonía con las tendencias en sostenibilidad como por ejemplo la eliminación de plásticos de un solo uso y la responsabilidad extendida del productor.

En síntesis, el papel del diseñador está en saber interpretar más allá de los aspectos técnicos los valores y potencialidades del material, y de facilitar la transición a procesos que garanticen la viabilidad y transferencia en el aprovechamiento, conformación y aplicación de materiales alternativos, a través de su exploración y experimentación. 


\section{REFERENCIAS BIBLIOGRÁFICAS}

Ashby, M., \& Johnson, K. (2014). In Elsevier Ltd. (Ed.), Materials and design (3rd ed.). Reigate, Surrey: Elsevier Science. Recuperado de http://www.sciencedirect.com/science/article/pii/B9780080982052000111

Ayala, C., \& Rognoli, V. (2015 ). DIY materials - the materials generation. Recuperado de http://www. diymaterials.it/

Ballesteros, M. \& Beltrán Luengas, E.M. (2018) ¿Investigar Creando? Una guía para la investigación-creación en la academia. Bogotá:Universidad El Bosque

Bistagnino, L. (2009). Design sistemico. progettare la sostenibilità produttiva e ambientale. Bra, Italia: Slow Food.

Centro de materiales para la innovación de Bogotá - Región. (2019). "Materiales: Oportunidades de innovación" . Bogotá: Cámara de Comercio de Bogotá, Universidad Jorge Tadeo Lozano, Secretaría Distrital de Desarrollo Económico.

Colombia productiva. (2020). Guía empresarial de economía circular. Colombia: Fiduciaria Colombiana de Comercio Exterior S.A. -FIDUCOLDEX. Recuperado de https://www.colombiaproductiva.com/ptp-capacita/ publicaciones/transversales/guia-empresarial-de-economia-circular/200310-cartilla-economia-circular

Ellen Macarthur Foundation. (S.f.). What is the circular economy? Recuperado de https://www.ellenmacarthurfoundation.org/circular-economy/what-is-the-circular-economy

Gobierno de la República de Colombia, 2019. Estrategia nacional de economía circular. Cierre de ciclos de materiales, innovación tecnológica, colaboración y nuevos modelos de negocio. Bogotá D.C., Colombia. Presidencia de la República; Ministerio de Ambiente y Desarrollo Sostenible; Ministerio de Comercio, Industria y Turismo. 
IDEO - Ellen Macarthur Foundation. (2016). The circular design guide. Recuperado de https://www. circulardesignguide.com/

Karana, E., Barati, B., Rognoli, V., \& Zeeuw van der Laan, Anouk. (2015). Material driven design (MDD): A method to design for material experiences. International Journal of Design, in press

Mateus Guerra, A. M., \& Buraglia Osorio, M., directora. (2019). Egglast sistema de empaque biodegradable para comida tipo B.A.R.F; (tesis de pregrado). Universidad El Bosque. Facultad de Creación y Comunicación. Programa de Diseño Industrial.

Mcdonough, W., \& Braungart, M. (2005). Cradle to cradle = de la cuna a la cuna: Rediseñando la forma en que hacemos las cosas. España: S.A. Mcgraw-Hill / Interamericana de España.

Merchan Camelo, M., \& Buraglia Osorio, M., directora. (2019). Ámbar el amor tiene forma de mango; (tesis de pregrado de artes y diseño) Universidad El Bosque. Facultad de Creación y Comunicación. Programa de Diseño Industrial.

Nuncira Rincón, A., Buraglia Osorio, M. a., \& Gómez Puertas, Sandra Rocio asesor. (2018). Suamne : Cubiertos y empaques en bagazo de caña para la reforestación (tesis de pregrado de artes y diseño) Universidad El Bosque. Facultad de Creación y Comunicación. Programa de Diseño Industrial.

Organización de Naciones Unidas [ONU], 2015. GOAL 12: Sustainable consumption and production: Data and statistics / Facts and figures. Recuperado de https://www.unep.org/explore-topics/sustainable-development-goals/ why-do-sustainable-development-goals-matter/goal-12

Ramos Riascos, C. D., \& Caro Vives, J. C. (2014). Biocompuestos en Colombia. aportes de diseño en la aplicación de biocompuestos en el contexto productivo colombiano. MasD Revista Digital De Diseño, 8(14), 1-8. Recuperado de https://masd.unbosque.edu.co/article/view/D01/pdf\#: :text=Un\%20 bio\%2Dcompuesto\%20es\%20un,en\%20su\%20proceso \%20de\%20producci\%C3\%B3n.

Rognoli, V., Bianchini, M., Maffei, S., \& Karana, E. (2015). DIY materials. Materials and Design, 86, 692-702. doi:10.1016/j.matdes.2015.07.020

Tecnalia, \& Departamento Nacional de Planeacion. (2018). Transición a una economía circular en colombia para el sector de la manufactura y de la construcción. Plan de acción recomendado. . (). Bogota, Colombia: Recuperado de https:/www.dnp.gov.co/Crecimiento-Verde/Documents/ejes-tematicos/Circular/ MATEC\%20Producto\%203.pdf

Wong, W. (2014). Fundamentos del diseño. Barcelona: Editorial Gustavo Gili. Recuperado de https://elibro. net/ereader/elibrodemo/45553 